\title{
Paradoxes in a Multi-criteria Routing Game
}

\author{
Amina Boukoftane ${ }^{1}$, Eitan Altman $^{2,3}$, Majed Haddad $^{4}$, and Nadia Oukid ${ }^{1}$ \\ University of Blida 1, Route de Soumaa, B.P. 270, Blida, Algeria ${ }^{1}$ \\ Universit Cote d'Azur (UCA), INRIA, 06902 Sophia-Antipolis, France ${ }^{2}$ \\ LINCS, 23 avenue d'Italie, 75013 Paris, France ${ }^{3}$ \\ LIA/CERI, University of Avignon, Agroparc, BP 1228, 84911, Avignon, France ${ }^{4}$
}

\begin{abstract}
In this paper, we consider a routing game in a network that contains lossy links. We consider a multi-objective problem where the players have each a weighted sum of a delay cost and a cost for losses. We compute the equilibrium and optimal solution (which are unique). We discover here in addition to the classical Kameda type paradox another paradoxical behavior in which higher loss rates have a positive impact on delay and therefore higher quality links may cause a worse performance even in the case of a single player.
\end{abstract}

Keywords: Routing game, multi-objective problem, lossy links, Nash equilibrium, price of anarchy, paradox.

\section{Introduction}

There has been much work on routing games with additive costs (cost associated with a route is additive over the links of the route) [6,7]. This has been extended to multi-objective additive criteria, see e.g., $[4,8,6]$. Little is known however on routing games with non additive costs. There has been some work on routing games for some given simple topologies with non-additive costs [6] triggered by networking applications (e.g., $[5,3])$.

In this work, we consider costs related to weighted sum of two different types of performances: the delay which is additive, and losses which are not. We focus on a simple network model that has been studied in the case of a single objective by $[2,3]$. We first derive explicit expression of the equilibrium and then study numerically its properties.

In case of a single objective, it has been shown that a Braess type paradox exists for the topology that we consider [2,3]. We identify a new type of paradox which has some surprising behavior. We then compute the price of anarchy (defined as in [1]).

The rest of the paper is organized as follows: In Section 2, we describe the system model and the performance measures adopted throughout the paper. In Section 3, we compute the global optimum. The Nash equilibrium is computed in Section 4. Simulation results along with the discussion are presented in Section 6 . Performance results including the price of anarchy and paradoxes are provided in 5 . Section 7 concludes the paper. 


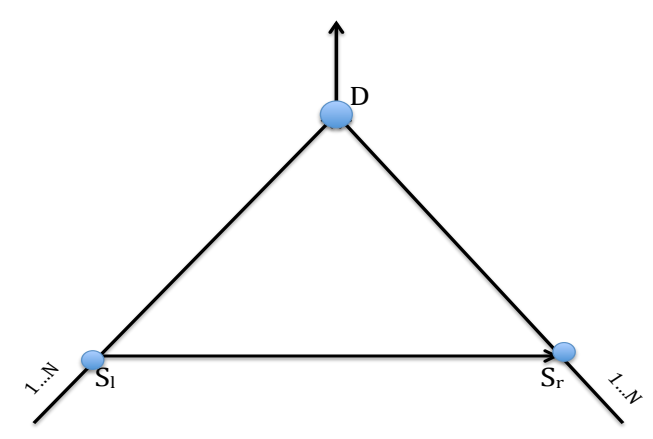

Fig. 1. The competitive routing model.

\section{The model and performance measures}

We shall use the load balancing network topology introduced in [2] consisting of three nodes: two source nodes $S_{r}$ and $S_{l}$ ( $r$ stands for right and $l$ for left) and one common destination node $D$ (see Figure 1). There are $2 N$ sources of flows, $S(i), i=1, \ldots, 2 N$. Each flow consists of an independent Poisson distributed point process with a rate $\phi$. Packets from source $i=1, \ldots, N$ arrive packets from source $i=N+1, \ldots, 2 N$ arrive at node $S_{r}$ (right). Source $i=1, \ldots, N$ can split its flow between its direct path $S_{l} D$ and the indirect one $S_{l} S_{r} D$. Source $i=N+1, \ldots, 2 N$ can split its flow between its direct path $S_{r} D$ and the indirect one $S_{r} S_{l} D$.

More precisely, whenever a packet arrives from source $i$, the source flips a coin that has a probability $p_{i}$ to have an outcome called "direct" and a probability of $1-p_{i}$ to have an outcome called "indirect". If the outcome is "direct" then the packet is routed over the direct route, and otherwise it is routed over the indirect one. The process of packets originating from source $i$ that take the direct path is thus Poisson with rate $\phi p_{i}$. The process of packets that arrive at node $i$ and that take the indirect path is Poisson with rate $\phi\left(1-p_{i}\right)$. Let $x_{l}^{i}$ be the rate of flow sent by source $i$ through link $l$. Links $S_{r} S_{l}$ and $S_{l} S_{r}$ are assumed to be wireless so that packets sent over $S_{r} S_{l}$ and $S_{l} S_{r}$ suffer independent losses with some fix probability $q$. The delay over these links is assumed to be a constant denoted by $\delta$. Links $S_{l} D$ and $S_{r} D$ are assumed to be lossless but they incur a congestion cost per flow unit that uses them of $T_{l}(x)=1 /\left(C-x_{l}\right)$. Here, $C$ is the link capacity and $x_{l}$ is the total flow going through link $l$. Therefore for each player $i$, consider the arrival process of packets that arrive at node $S_{k}$ and that are rerouted to the indirect path $S_{k} S_{m} D$ (where $k=l, r, m \neq k$ ). It is a Poisson process as well and its rate is $\phi p\left(1-p_{i}\right)(1-q)$. 
Let $i \leq N$. The cost for source $i$ is a weighted sum of the average delay of its flow and its loss rate:

$$
\begin{gathered}
J_{i}(p)=\frac{\phi p_{i}}{C-\phi \sum_{j=1}^{N} p_{j}-\phi q \sum_{i=N+1}^{2 N}\left(1-p_{j}\right)}+ \\
\frac{\phi(1-q)\left(1-p_{i}\right)}{C-\phi \sum_{j=N+1}^{2 N} p_{j}-\phi q \sum_{i=1}^{N}\left(1-p_{j}\right)}+ \\
\phi \delta(1-q)\left(1-p_{i}\right)+\gamma \phi q\left(1-p_{i}\right) .
\end{gathered}
$$

The three first terms correspond to the delay cost and the last term corresponds to the cost of losses. The first term corresponds to the congestion cost in the direct path of $i$ and the two following terms correspond to the congestion cost along the indirect path. The optimal symmetric solution is obtained by minimizing $\sum_{i} J_{i}(p)$ over $p_{i}$ and adding the constraint that $p_{i}$ are the same for all $i$ (we then omit $i$ from the notation $p_{i}$ ).

Let

$$
X_{l}(x)=\phi \sum_{j=1}^{N} p_{j}+\phi q \sum_{j=N+1}^{2 N}\left(1-p_{j}\right)
$$

be the rate of packets that use link $S_{l} D$. We assume that the link cost per unit of flow are linear in the flow through them. This amounts to use the first term in the Taylor's expansion of $(1) . J_{i}(p)$ is thus approximated by

$$
\begin{gathered}
J_{i}(p)= \\
\phi p_{i}\left(a x_{l}+b\right)+\phi(1-q)\left(1-p_{i}\right)\left(a x_{l}+b\right)+\delta \phi\left(1-p_{i}\right)+\gamma \phi q\left(1-p_{i}\right)
\end{gathered}
$$

where $a$ and $b$ are some positive constants.

\section{Global optimum calculation}

The global optimal solution is obtained by solving

$$
\frac{\partial}{\partial p} \sum_{i=1}^{2 N} J_{i}(p)=0
$$

(unless it is on the boundary). We obtain the unique solution:

$$
p=\frac{1}{2} \frac{a \phi N\left(2 q^{2}-2 q+1\right)+b q-\gamma q-\delta}{\phi a q N(q-1)} .
$$

We find it convenient in the numerical investigation to write $z=\gamma q+\delta$ since the dependence of the global optimum or the equilibrium on each one of the two parameters $\delta$ and $\gamma$ (for fixed $q$ ) appears only through the value of $z$. Thus, $p$ can be written: 


$$
p=\frac{1}{2} \frac{a \phi N\left(2 q^{2}-2 q+1\right)+b q-z}{\phi a q N(q-1)} .
$$

Note that

$$
J^{\prime}(p)=2 a \phi^{2} q^{2} N(1-p)+\phi b q+\phi^{2} a N+2 a \phi^{2} q N(p-1)-\phi z .
$$

$J^{\prime}(p)=0$ for

$$
z=-2 a \phi q N(((1-p)(1-q))+b q+a \phi N
$$

$J^{\prime}(p)$ is positive if $z<\phi a N(2 q(q-1)(1-p)+1)+q b$. In this case $J$ is an increasing function, and the minimum is reached on 0 .

On the other hand, if $z>\phi a N(2 q(q-1)(1-p)+1)+q b, J$ is a decreasing function, and the minimum is reached on 1 .

Note that

$-p<1$ if $z<a \phi N+q b$

$-p>0$ if $z>a \phi N\left(2 q^{2}-2 q+1\right)+q b$.

\section{Equilibrium calculation}

The equilibrium is obtained by setting $p_{i}=p$ to be the same for all $i$ except for $i=1$ where it is taken to be equal to $\hat{p}$. We then find for each value $p$ the best response $\hat{p}=f(p)$ for player 1. A fixed point of this equation provides the equilibrium. We did the same as below and get the Nash equilibrium which is equal to:

$$
p_{1}=\frac{1}{2} \frac{-a \phi q N(p+q-p q)+q(\phi a p+\phi a-b+\gamma)-\phi a+\delta}{\phi a q} .
$$

Let $\hat{p}$ be the point obtained by replacing $p_{1}$ by $p$, we obtain:

$$
\begin{gathered}
\hat{p}=\frac{a \phi N q^{2}+a \phi(1-q)+q b-\gamma q-\delta}{a \phi q(q N-N-1)} . \\
\hat{p}=\frac{a \phi N q^{2}+a \phi(1-q)+q b-z}{a \phi q(q N-N-1)} .
\end{gathered}
$$

We have $q N<N<N+1$, so the denominator is negative.

By differentiating the cost function with respect to $\hat{p}$ and setting the derivative equal to zero, we get for equilibrium these conditions:

$-J$ is an increasing function if: $z>2 a \phi q N-a \phi N+b q$,

$-J$ is a decreasing function if: $z<2 a \phi q N-a \phi N+b q$.

Also note that

$-\hat{p}<1$ if $z<a \phi(q N+1)+b q$

$-\hat{p}>0$ if $z>a \phi q^{2} N+a \phi(1-q)+b q$ 


\section{$5 \quad$ Price of anarchy and paradoxes}

The price of anarchy is the ratio of the worst case objective function value of a Nash equilibrium and that of an optimal outcome. That measures how the efficiency of a system degrades due to selfish behavior of its agents. The price of anarchy [1] is a method to measure the inefficiency of equilibrium, it has been used to measure the inefficiency in congestion networks. In this case, each user of the network has a source and destination and they must pay a cost to travel from their source to their destination. In this case it is given by:

$$
\operatorname{Po} A=\frac{2 N J_{i}(\hat{p})}{2 N J_{i}(p)}
$$

We say that a paradox occurs if when replacing links with higher quality ones result in worse performance. In our case, a higher quality link could mean a link with smaller delay $\delta$ or one with a smaller loss probability $q$. For example, there is a paradox if the derivative $J^{\prime}(\delta)$ of $J$ at the equilibrium w.r.t. the delay $\delta$ is negative, where

$$
\begin{gathered}
J^{\prime}(\delta)= \\
\frac{a \phi+a \phi N^{2}\left(1+q^{2}\right)+2 a \phi N q(1-N)+2 b q-2 \delta-2 \gamma q}{a q(q N-N-1)^{2}}
\end{gathered}
$$

In a similar network with a single objective for each player, a paradoxes been observed $[5,3,2]$ in which, for suitable parameters, improving the quality of the $\operatorname{link}(\mathrm{s})$ between $S_{r}$ and $S_{l}$ results in worse performance for all players. We may search for a similar paradox in our problem in which the quality of the link stands for its delay (higher quality means lower delay) or loss rate (higher quality means lower loss rate). The condition for this type of paradox is then that $J$ at equilibrium would be decreasing in the network parameter (e.g., in the delay $\delta$ ). Thus, the derivative of $J^{\prime}$ at equilibrium should be decreasing where the latter is given by

$$
J^{\prime}(\delta)=\frac{a \phi+a \phi N^{2}\left(1+q^{2}\right)+2 a \phi N q(1-N)+2 b q-2 z}{a q(q N-N-1)^{2}}
$$

Note that $J$ is a decreasing function if

$$
z>\frac{1}{2} \phi a\left(1+N^{2}\right)-\frac{1}{2} \phi a q N(2 N-q N-2)+b q
$$

\section{$6 \quad$ Numerical results}

Let us now validate our theoretical findings through numerical simulations. We consider $a=1, b=1, N=4, \phi=1, q=0.5$. Notice that the domain of existence of $z$ in this case is $[2.2,3]$

Fig. 2 depicts the equilibrium $\hat{p}$ and the optimal solution $p$ as function of $z$. As expected, we observe that both $\hat{p}$ and $p$ are increasing in $z$, while the equilibrium 


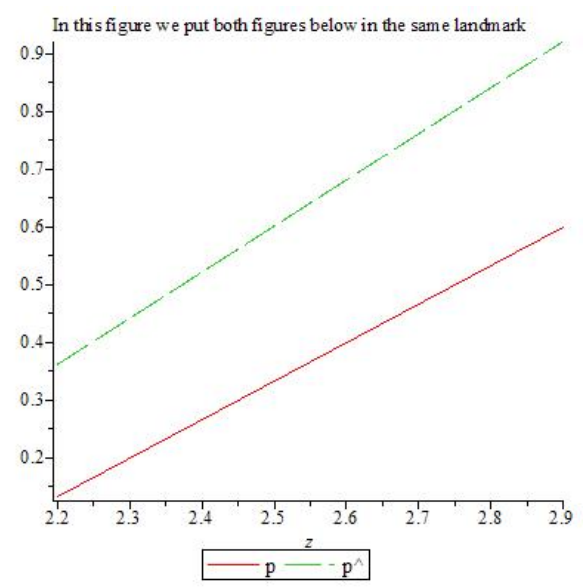

Fig. 2. The optimal solution and the equilibrium as a function of $z$.

$\hat{p}$ dominates the optimal solution $p$. Fig. 3 depicts the cost function of the optimal solution $J(p)$ and the equilibrium $J(\hat{p})$ as a function of $z$. We observe that both curves are increasing functions. In Fig. 4, we present respectively the variation of the cost function at the optimal solution and at the equilibrium as a function of the loss probability $q$. Fig. 5 depicts the variation of the cost function at the equilibrium as a function of the link delay $\delta$. The price of anarchy is presented in Fig. 6. As expected, for low values of $z$, the price of anarchy tends to 1.

\section{Discussion}

New paradox: We identify in Figure 4 a new type of paradox: the cost is seen not to be monotone in the quality of the link (the loss probability $q$ ). This phenomenon is due to the particular multi-objective structure of our problem. Indeed, higher $q$ increases the cost related to losses, but contributes to decreasing the global cost as more losses results in lower congestion and thus in lower delays. Kameda-paradox: We obtain in Figure 5 the paradox already observed in $[5,3,2]$ in which larger link delay are beneficial for all users. Investing in faster links increases the delay and deteriorates the performance for all players.

\section{Conclusion}

In this paper, we have studied the routing game with lossy links and congestion. The cost included both a delay component as well as one corresponding to the losses. After computing the unique optimal solution and the symmetric equilibrium, we have showed that even in the case of global optimization there may be a paradox due to the fact that increasing the loss rate may be advantageous when delays are high. In addition the Kameda-paradox has been also shown to occur here. 


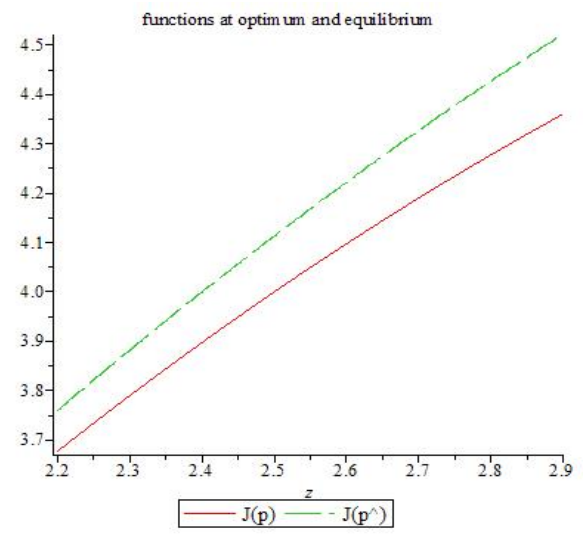

Fig. 3. The cost function at the optimal solution and the equilibrium as a function of $z$.

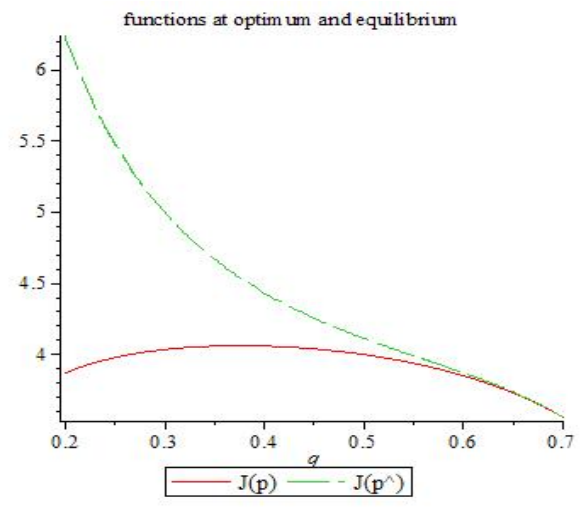

Fig. 4. The cost function $J$ as a function of the loss probability $q$.

\section{References}

1. E. Koutsoupias and C. Papadimitriou. Worst-case equilibria. In Proc. STACS, 1999.

2. H. Kameda, E. Altman, T. Kozawa, and Y. Hosokawa, Braess-like paradoxes in distributed computer systems. IEEE Trans. on Automatic Control 45 (9) 1687 1690 (2000).

3. E. Altman, J. Kuri, R. El-Azouzi. A routing game in networks with lossy links.7th International Conference on NETwork Games Control and OPtimization (NETGCOOP 2014), Oct 2014, Trento, Italy. 2014.

4. Yunan Wu, Yuchen Peng, Long Peng, Ling Xu "Super Efficiency of Multicriterion Network Equilibrium Model and Vector Variational Inequality", Journal of Optimization Theory and Applications, Volume 153, Issue 2, pp 485-496, May 2012.

5. E. Altman, R. El-Azouzi and V. Abramov , "Non-Cooperative Routing in Loss Networks" , Performance Evaluation, Vol 49, Issue 1-4, pp. 43-55, 2002. 


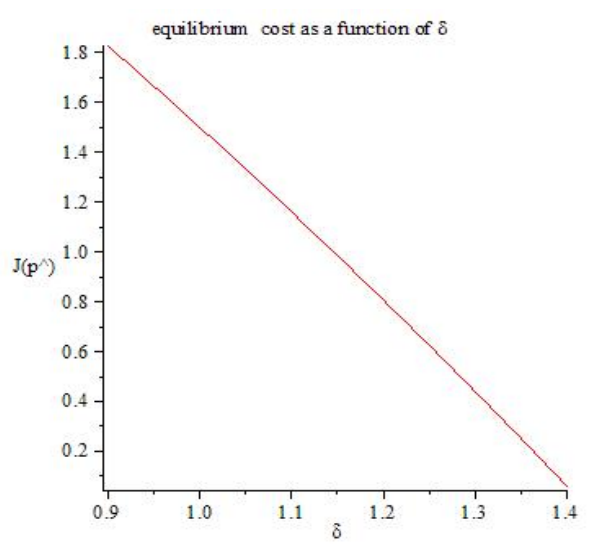

Fig. 5. $\mathrm{J}(\hat{p})$ as a function of the link delay $\delta$.

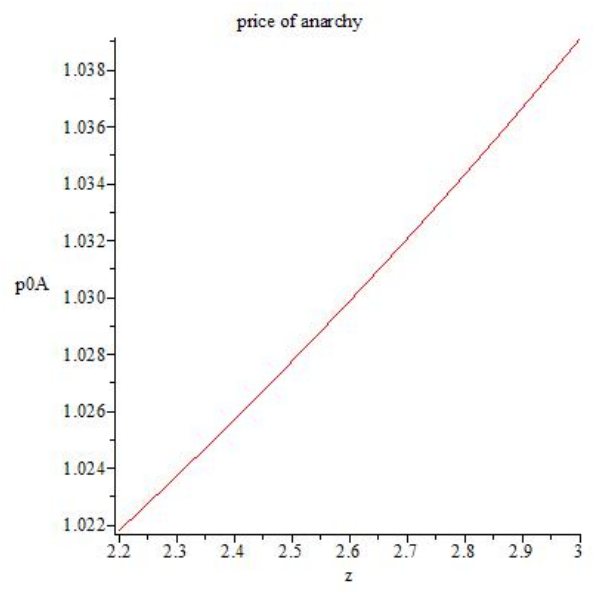

Fig. 6. The price of anarchy.

6. Altman, T. Boulogne, R. El Azouzi, T. Jimenez and L. Wynter, "A survey on networking games in telecommunications"; Computers and Operations Research, 33, pp 286-311, 2006.

7. L. Wynter and E. Altman, "Equilibrium, games, and pricing in transportation and telecommunications networks", Networks and Spacial Economics, special issue of on "Crossovers between Transportation Planning and Telecommunications", Vol. 4, Issue 1, p. 7-21, March 2004.

8. El-Azouzi R, Altman E. "Constrained traffic equilibrium in routing", IEEE Transactions on Automatic Control 2003;48(9):1656-1660. 